Bull. Korean Math. Soc. 49 (2012), No. 6, pp. 1349-1357

http://dx.doi.org/10.4134/BKMS.2012.49.6.1349

\title{
ON FOURIER COEFFICIENTS OF SOME MEROMORPHIC MODULAR FORMS
}

\author{
Yutaro Honda And Masanobu KaneKo
}

ABSTRACT. We prove a congruence modulo a prime of Fourier coefficients of several meromorphic modular forms of low weights. We prove the result by establishing a generalization of a theorem of Garthwaite.

\section{Main theorem}

For integers $k \geq 0$ and $N \geq 1$, let $M_{k}\left(\Gamma_{0}(N)\right)$ (resp. $\left.S_{k}\left(\Gamma_{0}(N)\right)\right)$ be the space of holomorphic modular (resp. cusp) forms of weight $k$ on the standard congruence subgroup $\Gamma_{0}(N)$ of the modular group $S L_{2}(\mathbb{Z})$. Let

$$
E_{k}(z)=1-\frac{2 k}{B_{k}} \sum_{n=1}^{\infty}\left(\sum_{d \mid n} d^{k-1}\right) q^{n} \quad\left(B_{k}: \text { the Bernoulli number, } q=e^{2 \pi i z}\right)
$$

be the normalized Eisenstein series of weight $k$ for $S L_{2}(\mathbb{Z})$ and

$$
\eta(z)=q^{1 / 24} \prod_{n=1}^{\infty}\left(1-q^{n}\right)
$$

the Dedekind eta function. For a prime $p$ and a modular form $f(z)=\sum c(n) q^{n}$, we consider the $U(p)$-operator defined by

$$
(f \mid U(p))(z):=\sum c(p n) q^{n} .
$$

We denote by $\mathbb{Z}_{(p)}$ the set of rational numbers whose denominators are prime to $p$.

In the present paper, we prove the following theorem.

Theorem 1. 1) For $k \in\{4,6,8,10,14\}$ and any prime $p$ with $p \equiv 1(\bmod 3)$, we have

$$
\frac{E_{k}(6 z)}{\eta(6 z)^{4}} \mid U(p) \equiv 0 \quad(\bmod p) .
$$

Received July 7, 2011; Revised November 7, 2011.

2010 Mathematics Subject Classification. 11F30, 11F33.

Key words and phrases. meromorphic modular form, congruence of Fourier coefficients, congruence subgroup. 
Here, the congruence means every Fourier coefficient on the left-hand side is divisible by $p$.

2) For $k \in\{4,6\}$, any prime $p \equiv 1(\bmod 4)$, and any modular form $f(z) \in$ $M_{k}\left(\Gamma_{0}(2)\right) \cap \mathbb{Z}_{(p)}[[q]]$ having p-integral rational Fourier coefficients, we have

$$
\frac{f(4 z)}{\eta(4 z)^{2} \eta(8 z)^{2}} \mid U(p) \equiv 0 \quad(\bmod p) .
$$

3) For any prime $p \equiv 1(\bmod 3)$ and any modular form $f(z) \in M_{4}\left(\Gamma_{0}(3)\right) \cap$ $\mathbb{Z}_{(p)}[[q]]$, we have

$$
\frac{f(3 z)}{\eta(3 z)^{2} \eta(9 z)^{2}} \mid U(p) \equiv 0 \quad(\bmod p) .
$$

4) For any prime $p \equiv 1(\bmod 3)$ and any modular form $f(z) \in M_{4}\left(\Gamma_{0}(4)\right) \cap$ $\mathbb{Z}_{(p)}[[q]]$, we have

$$
\frac{f(3 z)}{\eta(6 z)^{4}} \mid U(p) \equiv 0 \quad(\bmod p) .
$$

We prove the theorem by establishing a generalization of a theorem of Sharon Garthwaite [1], which we state and prove in the next section where we prove the theorem. In the last section we give some conjectures concerning congruences modulo higher powers of $p$, as well as the motivation of this work.

We should like to thank Ken Ono for showing us the proof of 1) of the theorem by using the original theorem of Garthwaite. Also our thanks go to Pavel Guerzhoy, who made substantial progress concerning our conjecture using the theory of weak harmonic Maass forms and whose interest gave us a strong impetus to write up the present paper.

\section{Proof}

To prove Theorem 1, we use the following theorem. The case of $N=1$ is due to Garthwaite [1, Theorem 1.3].

Theorem 2. Fix a prime number $p \geq 5$. For a natural number $N \in\{1,2,3,4\}$, let $a$ and $b$ be integers satisfying

$$
1 \leq a \leq b-2, \quad b \in \begin{cases}\{4,6,8,10,14\}, & \text { if } N=1, \\ \{4,6\}, & \text { if } N=2, \\ \{4\}, & \text { if } N=3,4,\end{cases}
$$

and set $k=a(p-1)+b$. Suppose $f(z) \in S_{k}\left(\Gamma_{0}(N)\right) \cap \mathbb{Z}_{(p)}[[q]]$. Then we have

$$
(f \mid U(p))(z) \equiv 0 \quad(\bmod p) .
$$

Proof. We give a somewhat simpler proof of the case $N=1$. The point is to construct an appropriate auxiliary function, and once it is found, the argument in other cases of $N=2,3,4$ is completely parallel. 
Suppose

$$
f(z)=\sum_{n=0}^{\infty} c(n) q^{n} \in S_{k}\left(\Gamma_{0}(1)\right) \cap \mathbb{Z}_{(p)}[[q]] \quad\left(\Gamma_{0}(1)=S L_{2}(\mathbb{Z})\right) .
$$

We prove the theorem (when $N=1$ ) by induction on $n$. Since $f(z)$ is a cusp form, we have $c(0)=0$. Let $n \geq 1$ and assume $c(p m) \equiv 0(\bmod p)$ for all $m$ with $0 \leq m \leq n-1$. We introduce the following function $g_{n}(z)=g_{n}^{(1)}(z)$;

$$
g_{n}(z):=\frac{E_{4}(z)^{3 p(n-1)} E_{14-b}(z)^{p-1} E_{p-1}(z)^{b-a-2}}{E_{b}(z)} .
$$

This is a meromorphic modular form of weight

$$
12(p n-1)-a(p-1)-b=12(p n-1)-k .
$$

We claim that the product $f(z) g_{n}(z)$ is a holomorphic modular form. The proof is as follows. The weight of $f(z) g_{n}(z)$ is $12(p n-1)$, a multiple of 12 . The possible poles of $f(z) g_{n}(z)$ come from the denominator $E_{b}(z)$ of $g_{n}(z)$ which has zeros of order at most 2 at points equivalent to $e^{2 \pi i / 3}$ and at most 1 at points equivalent to $\sqrt{-1}$. On the other hand, by the well-known valence formula, the orders of pole of $f(z) g_{n}(z)$ (of weight a multiple of 12) at $e^{2 \pi i / 3}$ is a multiple of 3 and at $\sqrt{-1}$ a multiple of 2 . Therefore the function $f(z) g_{n}(z)$ cannot have poles and is a holomorphic modular form of weight $12(p n-1)$. Hence the function

$$
\frac{f(z) g_{n}(z)}{\Delta(z)^{p n-1}}
$$

where $\Delta(z)=\eta(z)^{24}$ is the cusp form of weight 12 , is of weight 0 and holomorphic on the upper half-plane, and thus is a polynomial in the elliptic modular function

$$
j(z)=\frac{E_{4}(z)^{3}}{\Delta(z)} .
$$

We conclude then that the function

$$
\frac{f(z) g_{n}(z)}{\Delta(z)^{p n-1}}\left(-j^{\prime}(z)\right) \quad\left(j^{\prime}(z)=\frac{1}{2 \pi i} \frac{d}{d z} j(z)\right)
$$

is a derivative of a polynomial in $j(z)$ and thus the constant term of the Fourier expansion of

$$
\frac{f(z) g_{n}(z)}{\Delta(z)^{p n-1}}\left(-j^{\prime}(z)\right)=\frac{f(z) E_{4}(z)^{3 p(n-1)} E_{14-b}(z)^{p} E_{p-1}(z)^{b-a-2}}{\Delta(z)^{p n}}
$$

(note $E_{b}(z) E_{14-b}(z)=E_{14}(z)$ and $\left.-j^{\prime}(z)=E_{14} / \Delta(z)\right)$ vanishes. Write

$$
\frac{E_{4}(p z)^{3(n-1)} E_{14-b}(p z)}{\Delta(p z)^{n}}=q^{-p n}+\sum_{l=-n+1}^{\infty} a(p l) q^{p l} .
$$


Then we have

$$
\begin{aligned}
0 & =\text { constant term of } \frac{f(z) E_{4}(z)^{3 p(n-1)} E_{14-b}(z)^{p} E_{p-1}(z)^{b-a-2}}{\Delta(z)^{p n}} \\
& \equiv \text { constant term of } \frac{f(z) E_{4}(p z)^{3(n-1)} E_{14-b}(p z)}{\Delta(p z)^{n}}(\bmod p) \\
& \equiv \text { constant term of }(f \mid U(p))(p z)\left(q^{-p n}+\sum_{l=-n+1}^{\infty} a(p l) q^{p l}\right)(\bmod p) \\
& \equiv \text { constant term of }\left(c(p n) q^{p n}+\sum_{l=n+1}^{\infty} c(p l) q^{p l}\right)\left(q^{-p n}+\sum_{l=-n+1}^{\infty} a(p l) q^{p l}\right)(\bmod p) \\
& \equiv c(p n) \quad(\bmod p) .
\end{aligned}
$$

We have used the well-known congruence $E_{p-1}(z) \equiv 1(\bmod p)$. This establishes the case $N=1$ by induction.

For $N=2$, we put

$$
g_{n}^{(2)}(z):=\frac{E_{4}^{(2)}(z)^{p(n-1)} E_{6-b}^{(2)}(z)^{p} E_{p-1}(z)^{b-a-2}}{E_{6}^{(2)}(z)},
$$

where we define the Eisenstein series (at infinity) $E_{k}^{(2)}(z)$ of even weight $k$ for $\Gamma_{0}(2)$ by

$$
E_{k}^{(2)}(z):= \begin{cases}1, & k=0, \\ 2 E_{2}(2 z)-E_{2}(z), & k=2, \\ \frac{2^{k} E_{k}(2 z)-E_{k}(z)}{2^{k}-1}, & k \geq 4 .\end{cases}
$$

Here, $E_{k}(z)$ is the Eisenstein series for $S L_{2}(\mathbb{Z})\left(E_{2}(z)=1-24 q-72 q^{2}-\cdots\right.$ is a "quasimodular" form, but $E_{2}^{(2)}(z)$ is modular). The weight of $g_{n}^{(2)}(z)$ is $4(p n-1)-k$. Since the zeros of $E_{6}^{(2)}(z)$ are at the cusp 0 and at $\rho_{2}=$ $(-1+\sqrt{-1}) / 2$ (and points equivalent to them), and are both simple, and since by the valence formula we know that the order of pole at $\rho_{2}$ of $f(z) g_{n}^{(2)}(z)$ (of weight $4(p n-1)$, a multiple of 4$)$ is even, we conclude that the form $f(z) g_{n}^{(2)}(z)$ is a holomorphic modular form. Let $\Delta^{(2)}(z)$ be defined by

$$
\Delta^{(2)}(z):=\frac{\eta(2 z)^{16}}{\eta(z)^{8}}\left(=q+8 q^{2}+28 q^{3}+64 q^{4}+126 q^{5}+\cdots\right) .
$$

This is a modular form of weight 4 on $\Gamma_{0}(2)$ having only simple zero at infinity. The function

$$
j^{(2)}(z):=\frac{E_{4}^{(2)}(z)}{\Delta^{(2)}(z)}
$$


is the "Hauptmodul" of $\Gamma_{0}(2)$ and the quotient

$$
\frac{f(z) g_{n}^{(2)}(z)}{\Delta^{(2)}(z)^{p n-1}}
$$

is a polynomial in $j^{(2)}(z)$. We argue as in the case $N=1$ by using $-j^{(2)}(z)^{\prime}=$ $E_{6}^{(2)}(z) / \Delta^{(2)}(z)$ to see the constant term of

$$
\frac{f(z) g_{n}^{(2)}(z)}{\Delta^{(2)}(z)^{p n-1}}\left(-j^{(2)}(z)^{\prime}\right)=\frac{f(z) E_{6-b}^{(2)}(z)^{p} E_{p-1}(z)^{b-a-2}}{\Delta^{(2)}(z)^{p n}}
$$

is 0 . The proof of the theorem using this by induction goes completely in the same manner.

For $N=3$, we put

$$
g_{n}^{(3)}(z):=\frac{E_{1}^{(3)}(z)^{p-1} \Delta_{0}^{(3)}(z)^{p(n-1)} E_{p-1}(z)^{2-a}}{E_{4}^{(3)}(z)},
$$

where $E_{1}^{(3)}(z), \Delta_{0}^{(3)}(z)$ and $E_{4}^{(3)}(z)$ are defined by

$$
\begin{aligned}
& E_{1}^{(3)}(z):=1+6 \sum_{n=1}^{\infty}\left(\sum_{d \mid n}\left(\frac{d}{3}\right)\right) q^{n}=1+6 q+6 q^{3}+6 q^{4}+12 q^{7}+\cdots \\
& \Delta_{0}^{(3)}(z):=\frac{\eta(z)^{9}}{\eta(3 z)^{3}}=1-9 q+27 q^{2}-9 q^{3}-117 q^{4}+216 q^{5}+\cdots \\
& E_{4}^{(3)}(z):=\frac{3^{4} E_{4}(3 z)-E_{4}(z)}{3^{4}-1}=1-3 q-27 q^{2}+159 q^{3}-219 q^{4}+\cdots
\end{aligned}
$$

the symbol $\left(\frac{d}{3}\right)$ being the Legendre character. Further, let $\Delta_{\infty}^{(3)}(z)$ and $j^{(3)}(z)$ be defined by

$$
\begin{aligned}
\Delta_{\infty}^{(3)}(z) & :=\frac{\eta(3 z)^{9}}{\eta(z)^{3}}=q+3 q^{2}+9 q^{3}+13 q^{4}+24 q^{5}+\cdots \\
j^{(3)}(z) & :=\frac{E_{1}^{(3)}(z)^{3}}{\Delta_{\infty}^{(3)}(z)}=\frac{1}{q}+15+54 q-76 q^{2}-243 q^{3}+\cdots
\end{aligned}
$$

The functions $E_{1}^{(3)}(z), \Delta_{0}^{(3)}(z)$ and $\Delta_{\infty}^{(3)}(z)$ are "Nebentypus" modular forms for $\Gamma_{0}(3)$ of weights 1,3 , and 3 respectively. Their even powers are modular for $\Gamma_{0}(3)$. The modular form $E_{4}^{(3)}(z)$ (of weight 4 ) has its zeros at the cusp 0 and $\rho_{3}=-1 / 2+\sqrt{-3} / 6$, both being simple. From this and the valence formula (if the weight is a multiple of 3 , the order at $\rho_{3}$ is divisible by 3 ), we conclude as before that, for a cusp form $f(z)$ of weight $a(p-1)+4$, the function

$$
\frac{f(z) g_{n}^{(3)}(z)}{\Delta_{\infty}^{(3)}(z)^{p n-1}}
$$


(which has no pole at the cusp 0) is a polynomial in $j^{(3)}(z)$ and by using $-j^{(3)}(z)^{\prime}=E_{1}^{(3)}(z) E_{4}^{(3)}(z) / \Delta_{\infty}^{(3)}(z)$ that the constant term of

$$
\frac{f(z) g_{n}^{(3)}(z)}{\Delta_{\infty}^{(3)}(z)^{p n-1}}\left(-j^{(3)}(z)^{\prime}\right)=\frac{f(z) E_{1}^{(3)}(z)^{p} \Delta_{0}^{(3)}(z)^{p(n-1)} E_{p-1}(z)^{2-a}}{\Delta_{\infty}^{(3)}(z)^{p n}}
$$

vanishes. The rest of the arguments is the same.

Finally, when $N=4$, we put

$$
g_{n}^{(4)}(z):=\frac{\Delta_{0}^{(4)}(z)^{p(n-1)}}{E_{4}^{(4)}(z)},
$$

where

$$
\Delta_{0}^{(4)}(z):=\frac{\eta(z)^{8}}{\eta(2 z)^{4}}=1-8 q+24 q^{2}-32 q^{3}+24 q^{4}-\cdots
$$

is weight 2 and

$$
E_{4}^{(4)}(z):=\frac{2^{4} E_{4}(4 z)-E_{4}(2 z)}{2^{4}-1}=1-16 q^{2}+112 q^{4}-448 q^{6}+\cdots
$$

is weight 4 modular form on $\Gamma_{0}(4)$. The form $E_{4}^{(4)}(z)$ has zeros only at cusps 0 and $-1 / 2$ which are simple, and so for any cusp form $f(z)$ of weight $a(p-1)+4$ the product $f(z) g_{n}^{(4)}(z)$ is a holomorphic modular form of weight $2(p n-1)$. With the weight 2 modular form

$$
\Delta_{\infty}^{(4)}(z):=\frac{\eta(4 z)^{8}}{\eta(2 z)^{4}}=q+4 q^{3}+6 q^{5}+8 q^{7}+13 q^{q}+\cdots
$$

having only zero at infinity, we consider the quotient

$$
\frac{f(z) g_{n}^{(4)}(z)}{\Delta_{\infty}^{(4)}(z)^{p n-1}}
$$

which is of weight 0 and has pole only at infinity, and hence a polynomial in the Hauptmodul

$$
j^{(4)}(z):=\frac{E_{2}^{(4)}(z)}{\Delta_{\infty}^{(4)}(z)},
$$

where $E_{2}^{(4)}(z):=\left(4 E_{2}(4 z)-E_{2}(z)\right) / 3$. We have $-j^{(4)}(z)^{\prime}=E_{4}^{(4)}(z) / \Delta_{\infty}^{(4)}(z)$ and, just as in the previous cases, the constant term of

$$
\frac{f(z) g_{n}^{(4)}(z)}{\Delta_{\infty}^{(4)}(z)}\left(-j^{(4)}(z)^{\prime}\right)=\frac{f(z) \Delta_{0}^{(4)}(z)^{p(n-1)}}{\Delta_{\infty}^{(4)}(z)^{p n}}
$$

is 0 . We can proceed in the same way as before and completes the proof of Theorem 2. 
Proof of Theorem 1. 1) For $k \in\{4,6,8,10,14\}$ and $p \equiv 1(\bmod 3)$, consider the form

$$
F_{k}(z):=E_{k}(z) \Delta(z)^{\frac{p-1}{6}}
$$

which is a cusp form with integer coefficients of weight $2(p-1)+k$ on $S L_{2}(\mathbb{Z})$. To this form we apply Theorem 2 to obtain

$$
F_{k}(z) \mid U(p) \equiv 0 \quad(\bmod p)
$$

and this is apparently equivalent to

$$
F_{k}(6 z) \mid U(p) \equiv 0 \quad(\bmod p) .
$$

On the other hand, we have

$$
\Delta(6 z)^{\frac{p-1}{6}}=\eta(6 z)^{4(p-1)} \equiv \frac{\eta(6 p z)^{4}}{\eta(6 z)^{4}} \quad(\bmod p) .
$$

We therefore have

$$
\frac{E_{k}(6 z)}{\eta(6 z)^{4}} \eta(6 p z)^{4} \mid U(p) \equiv 0 \quad(\bmod p) .
$$

This clearly implies

which is to be proved.

$$
\frac{E_{k}(6 z)}{\eta(6 z)^{4}} \mid U(p) \equiv 0 \quad(\bmod p)
$$

2) For $k \in\{4,6\}, p \equiv 1(\bmod 4)$, and $f \in M_{k}\left(\Gamma_{0}(2)\right) \cap \mathbb{Z}_{(p)}[[q]]$, consider the cusp form

$$
F_{k}^{(2)}(z):=f(z)\left(\eta(z)^{8} \eta(2 z)^{8}\right)^{\frac{p-1}{4}} .
$$

Here, $\eta(z)^{8} \eta(2 z)^{8}$ is the cusp form of weight 8 on $\Gamma_{0}(2)$. Applying Theorem 2 with the same argument as in 1), we obtain 2) of Theorem 1.

3) Here also the proof goes in the same manner by looking at the cusp form

$$
F_{k}^{(3)}(z):=f(z)\left(\eta(z)^{6} \eta(3 z)^{6}\right)^{\frac{p-1}{3}},
$$

where $\eta(z)^{6} \eta(3 z)^{6}$ is the cusp form of weight 6 on $\Gamma_{0}(3)$.

4) This case the form

$$
F_{k}^{(4)}(z):=f(z)\left(\eta(2 z)^{12}\right)^{\frac{p-1}{3}}
$$

plays the same role as previous cases and we are done.

\section{Remarks}

By computer calculations, we are tempted to pose the following.

Conjecture 1. Let $k \in\{4,6,8,10,14\}, p \equiv 1(\bmod 3)$ and

$$
F_{k}(z)=\frac{E_{k}(6 z)}{\eta(6 z)^{4}}
$$

as in Theorem 1. Then the congruence

$$
F_{k}(z) \mid U(p)^{m} \equiv 0 \quad\left(\bmod p^{m(k-3)}\right)
$$


holds for any integer $m$, and similarly for other cases $N=2,3,4$.

We may also expect that Theorem 2 could be extended to congruences of higher power of $p$, but this extension seems not enough to imply the above conjecture.

As mentioned in $\S 1, \mathrm{P}$. Guerzhoy [2] proved the conjecture for $k=6$ and "almost" proved for $k=4$ (with some defect in power), as well as other cases of $p$ not considered in this paper. He uses the theory of weak harmonic Maass forms and it seems the techniques there could imply various general results.

Finally, we mention our motivation of looking at those particular meromorphic modular forms in Theorem 1. In [3], we studied various modular (and quasimodular) solutions of the following differential equation (with a parameter $k$ ), which appeared in [4] in connection to supersingular $j$-invariants:

$$
f^{\prime \prime}(z)-\frac{k+1}{6} E_{2}(z) f^{\prime}(z)+\frac{k(k+1)}{12} E_{2}^{\prime}(z) f(z)=0 .
$$

This differential equation is also closely related to the two dimensional conformal field theory ( $c f$. e.g., [5]). For $k=4$, this equation has $E_{4}(z)$ as a solution and the other independent solution is given by

$$
E_{4}(z) \cdot \int_{z}^{i \infty} \frac{\Delta(z)^{5 / 6}}{E_{4}(z)^{2}} \frac{d z}{2 \pi i}
$$

which is apparently not modular. We were curious about the arithmetic nature of this solution, and found numerically that no primes of the form $3 n+1$ appear in the denominators of coefficients of $q^{(6 n+5) / 6}$ in $\Delta(z)^{5 / 6} / E_{4}(z)^{2}$. By the equation

$$
\frac{\Delta(z)^{5 / 6}}{E_{4}(z)^{2}}=-\frac{1}{3456} \frac{E_{4}(z)}{\eta(z)^{4}}-\frac{1}{576}\left(\frac{E_{6}(z)}{\eta(z)^{4} E_{4}(z)}\right)^{\prime} \quad\left({ }^{\prime}=q \frac{d}{d q}\right),
$$

this is equivalent to the statement in Theorem 1-1) with $k=4$. We naturally searched for other meromorphic modular forms having similar congruence property. There are many examples to which our proof given here does not apply.

\section{References}

[1] S. Garthwaite, Convolution congruences for the partition function, Proc. Amer. Math. Soc. 135 (2007), no. 1, 13-20.

[2] P. Guerzhoy, On the Honda-Kaneko congruences, preprint, 2011.

[3] M. Kaneko and M. Koike, On modular forms arising from a differential equation of hypergeometric type, Ramanujan J. 7 (2003), no. 1-3, 145-164.

[4] M. Kaneko and D. Zagier, Supersingular j-invariants, hypergeometric series, and Atkin's orthogonal polynomials, Computational perspectives on number theory (Chicago, IL, 1995), 97-126, AMS/IP Stud. Adv. Math., 7, Amer. Math. Soc., Providence, RI, 1998.

[5] S. Mathur, S. Mukhi, and A. Sen, On the classification of rational conformal field theory, Phys. Lett. B 213 (1988), no. 3, 303-308. 
ON FOURIER COEFFICIENTS OF SOME MEROMORPHIC MODULAR FORMS 1357

\author{
YutARO HoNDA \\ 5-12-18-503 \\ Nishitenma KitA-KU \\ OSAKA 530-0047, JAPAN \\ E-mail address: yu_ta_ro_honda@yahoo.co.jp \\ Masanobu KaneKo \\ FaCUlty of Mathematics \\ KYUSHU UNIVERSITY \\ FUKUOKA 819-0395, JAPAN \\ E-mail address: mkaneko@math.kyushu-u.ac.jp
}

